\title{
Consecuencias Clínicas de las Lesiones Cariosas no Tratadas y su Impacto en la Calidad de Vida de Preescolares Peruanos
}

\author{
Clinical Consequences of Untreated Carious Lesions and \\ Their Impact on the Quality of Life of Peruvian Preschoolers
}

Evelyn R. Munayco-Pantoja'; Héctor Pereyra-Zaldívar² \& Marleny M. Cadillo-lbarra ${ }^{3}$

MUNAYCO-PANTOJA, E. R.; PEREYRA-ZALDÍVAR, H. \& CADILLO-IBARRA, M. M. Consecuencias clínicas de las lesiones cariosas no tratadas y su impacto en la calidad de vida de preescolares peruanos. Int. J. Odontostomat., 15(1):256-262, 2021.

RESUMEN: La caries dental no tratada tiene repercusiones en la salud de los niños que la padecen, también repercute en el bienestar familiar; más aún, existe mayor probabilidad de presentar consecuencias clínicas, lo que agravaría la condición de salud. El objetivo del estudio fue investigar el impacto de las lesiones cariosas extensas en dentina y las consecuencias clínicas de lesiones cariosas no tratadas en la calidad de vida de preescolares peruanos. Un total de 150 niños, de 3 a 5 años, fueron evaluados usando ICDAS y pufa. También se evaluó la historia de extracción dental y se registró la presencia de dolor dental. El impacto de la salud oral en la calidad de vida fue evaluado mediante la versión peruana del ECOHIS (P-ECOHIS). Se utilizó el modelo de regresión logística binaria para analizar la asociación entre la prevalencia de lesiones cariosas extensas en dentina, pufa, historia de extracción y dolor dental con la puntuación del PECOHIS. La media del puntaje total del P-ECOHIS fue de 19,98 $\pm 6,9$, la prevalencia de las lesiones cariosas no tratadas y lesiones cariosas extensas en dentina fue de $90 \%$ y $71 \%$, respectivamente. El $77 \%$ de los dientes cariados no tratados desarrollaron una infección oral. El dolor dental tuvo una asociación estadísticamente significativa con el puntaje del PECOHIS $(p<0,05)(O R=3,76 ; 95 \%$ IC: 1,61 - 8,80). Los niños que presentaron compromiso pulpar (OR=2,44; $95 \%$ IC: 1,12 $-5,30)$ y presencia de fistula $(O R=4,39 ; 95 \%$ IC: $1,72-11,24)$ tuvieron altas probabilidades de tener altos puntajes en el $P$ ECOHIS $(p<0,05)$. Las lesiones cariosas extensas en dentina no impactan en la calidad de vida de los preescolares peruanos, como si impacta negativamente, el compromiso pulpar y la presencia de fistula como consecuencias clínicas de lesiones cariosas no tratadas.

PALABRAS CLAVE: caries dental, niño, calidad de vida, salud oral.

INTRODUCCIÓN

La caries dental sigue siendo una de las enfermedades más extendidas en el mundo (Petersen, 2009) y es un problema de salud pública que afecta a un gran porcentaje de niños (621 millones) (Kassebaum et al., 2015). En muchos países en desarrollo, el acceso a los servicios de salud oral es limitado y los dientes a menudo no se tratan (Petersen, 2003). La caries dental no tratada es la décima condición de salud más prevalente en niños preescolares (Nora et al., 2018) afectando 9,0 \% de la población infantil mundial (Peres et al., 2019) alcanzando su máximo valor entre niños de 1 a 4 años en el 2015 (Kassebaum et al., 2017).

Las etapas iniciales son asintomáticas, los niños y las familias tienden a experimentar menos impacto en la calidad de vida que aquellos con lesiones de caries moderadas y extensas (Piva et al., 2018). Los síntomas dolorosos comienzan después de que la lesión cariosa ha progresado a dentina (Selwitz et al., 2007). Los dien-

\footnotetext{
${ }^{1}$ Facultad de Odontología. Universidad Nacional Mayor de San Marcos. Lima, Perú. orcid.org/0000-0003-0417-262X.

2 Facultad de Medicina. Universidad Nacional Mayor de San Marcos. Lima, Perú. orcid.org/0000-0003-2449-4407.

${ }^{3}$ Instituto Nacional de Salud del Niño. Lima, Perú. orcid.org/0000-0003-4114-920X.
} 
MUNAYCO-PANTOJA, E. R.; PEREYRA-ZALDÍVAR, H. \& CADILLO-IBARRA, M. M. Consecuencias clínicas de las lesiones cariosas no tratadas y su impacto en la calidad de vida de preescolares peruanos. Int. J. Odontostomat., 15(1):256-262, 2021.

tes muy cariados tienen un impacto importante en la salud general, nutrición, crecimiento y peso de los niños (Duijster et al., 2013) al causar molestias, dolor, problemas de sueño, trastornos del aprendizaje y ausencia a la escuela (Seirawan et al., 2012). Si se extiende a pulpa, la caries trae como consecuencia, la infección odontogénica, esta es la razón más frecuente de hospitalización en niños pequeños (Nalliah et al., 2010) y en última instancia, los dientes se pierden prematuramente (Peres et al.). Es importante estudiar la severidad de las lesiones cariosas, ya que mientras más severa esta sea (profunda y extensa) habrá mayores probabilidades de tener consecuencias clínicas.

Las consecuencias de la caries dental no tratada en dientes deciduos y permanentes son registradas mediante el índice pufa/PUFA, publicada por Monse et al. (2010); este índice intenta complementar y aumentar la sensibilidad del índice de experiencia de caries ceod (cariado, extraído, obturado), ya que este no registra las consecuencias de una lesión cariosa (Mehta \& Bhalla, 2014). El índice pufa deriva de las primeras letras de cuatro afecciones orales resultantes de las caries no tratadas. Se calcula como la suma de cuatro diagnósticos de diferentes tipos de infecciones odontogénicas ([p] compromiso pulpar, [u] ulceración, [f] fístula y [a] absceso) (Monse et al.). Es importante estudiarlas por el impacto negativo que tienen en la calidad de vida relacionada a la salud oral de los niños (Corrêa-Faria et al., 2018).

La calidad de vida relacionada a la salud oral (CVRSB) se define como el impacto de las condiciones orales en el funcionamiento y bienestar diario (Locker \& Allen, 2007), tanto la caries dental no tratada activa e inactiva (Ramos-Jorge et al., 2014), grave o no (Nora et al.), en dientes anteriores (Clementino et al., 2015a) y posteriores ((Ramos-Jorge et al., 2015), y sus consecuencias clínicas impactan en la CVRSB de los niños en edad preescolar (Corrêa-Faria et al.). Esta información es considerada como una mejor forma de comunicación con los gestores de políticas públicas que simplemente el uso de medidas cuantitativas, como los índices de caries. Además de la posibilidad de crear conciencia en los padres y/o cuidadores (Allen, 2003). Lamentablemente, un análisis crítico de la literatura revela una escasez de estudios peruanos sobre la severidad de caries y consecuencias de caries dental no tratada. Por lo que el objetivo del presente estudio es investigar el impacto de las lesiones cariosas extensas en dentina y las consecuencias clínicas de lesiones cariosas no tratadas en la calidad de vida de preescolares peruanos.

\section{MATERIAL Y MÉTODO}

Los participantes del estudio fueron 150 niños y sus acompañantes, los cuales visitaron el departamento de odontología pediátrica del Instituto Nacional de salud del niño, en Lima - Perú, entre noviembre y diciembre del 2019. El permiso para realizar este estudio fue obtenido del Comité Institucional de Ética en Investigación del referido instituto (PI-08/19). El consentimiento escrito fue obtenido de los acompañantes previa entrevista, aplicación de la encuesta y evaluación oral.

\section{Criterios de selección de la muestra}

La muestra fue escogida por conveniencia por parte del investigador, se incluyeron niños, de ambos sexos, de 3 a 5 años de edad, que tengan por lo menos una lesión cariosa. Ellos debieron estar acompañados por su madre o padre. Los niños y acompañantes que hayan presentado enfermedades sistémicas o alguna discapacidad física o mental, no fueron considerados en el estudio, así como los acompañantes que no hayan firmado el consentimiento informado también fueron excluidos.

Recolección de datos. La recolección de datos fue realizada en dos partes; la primera parte fue la entrevista a los acompañantes, donde se obtuvo información socio-demográfica (ingreso familiar mensual y grado de instrucción). El ingreso familiar fue registrado como la remuneración mínima vital (RMV), según el estado peruano, esta es USD 273, También se obtuvo información sobre la historia de extracción de los niños y el dolor dental (si alguna vez se realizaron extracciones a los niños o experimentaron dolor por motivo de la caries dental no tratada).

Posteriormente, se realizaron las preguntas de la versión peruana de la escala de impacto de salud oral en preescolares (P-ECOHIS) validada por López Ramos et al. (2013), la cual consta de 13 preguntas, con 6 alternativas de respuesta: "nunca", "casi nunca", "ocasionalmente", "a menudo" y "muy a menudo" (equivalente a una puntuación de 0 a 4, respectivamente), la sexta alternativa de respuesta ("No sabe") no fue considerada en la investigación. Estas respuestas registran la frecuencia con la que ocurrió un evento durante la vida del niño (Arrow \& Klobas, 2015). El puntaje total oscila entre cero a 52 calculado como la suma de las respuestas. Una mayor puntuación denota una peor calidad de vida relacionada a la salud 
oral (CVRSB) y un mayor impacto en la salud oral (Ramos-Jorge et al., 2015).

La segunda parte fue la evaluación dental a los niños, previamente el investigador fue calibrado por un especialista experto en odontología pediátrica, mediante una preparación teórica (fotografías) y clínica, se evaluaron a 30 niños con la misma edad de la muestra, se utilizaron los índices ICDAS-II y pufa. Con respecto al índice ICDAS-II, los códigos C2 al C5 fueron registrados, pero solo se utilizó el código C6, para determinar las lesiones cariosas extensas en dentina (ICCMSTM Caries Management, 2020), el código $\mathrm{C} 1$ no fue utilizado por no contar con el aire de la jeringa triple de la unidad dental. Para determinar las lesiones cariosas no tratadas se utilizó desde el código C3 al C6.

Con respecto al índice pufa, con el cual se registraron las consecuencias de la caries dental no tratada, se utilizaron todos los códigos; $\mathrm{p}$ : compromiso pulpar, se observó la apertura de la cámara pulpar; u: ulceración por trauma, se observaron fragmentos de raíz que causaron ulceración traumática de los tejidos blandos circundantes; f: fístula, piezas con presencia de pus en el tracto sinusal y a: absceso, piezas con presencia de inflamación que contiene pus, ambos relacionados con un diente con afectación pulpar (Monse et al.). Solo se asignó un puntaje por diente, por lo que el puntaje pudo variar de 0 a 20. En caso de duda sobre el alcance de la infección odontogénica, se proporcionó la puntuación básica ( $p$ para la afectación pulpar). Se obtuvo una confiabilidad interexaminador de 0,80 , los participantes de la calibración no fueron incluidos en la muestra principal.

Para el examen clínico, se utilizó el espejo oral y la sonda periodontal de punta redondeada (sonda OMS), la cual se pasó suavemente sobre la superficie a examinar, para ICDAS-II; y para pufa, la evaluación se realizó visualmente sin el uso de un instrumento. Si el niño presentó placa bacteriana o restos de comida, se le indicó al acompañante realizarle la higiene respectiva antes de la evaluación.

Análisis de los datos. Los datos fueron analizados mediante el paquete estadístico SPSS versión 25 . La diferencia estadística en las puntuaciones del PECOHIS entre los que tuvieron impacto en la CVRSB de los que no la tuvieron, se evaluó mediante la prueba de chi cuadrado. Para esto, la puntuación del PECOHIS se dicotomizó utilizando la mediana (18) como punto de corte. El valor de $p$ fue $<0,05$, por lo que se consideró una diferencia estadísticamente significativa entre los dos grupos. El modelo de regresión logística binaria fue usado para evaluar la asociación entre la caries extensa en dentina (si/no), pufa $(0 \circ \geq 1)$, historia de extracción (si/no), dolor dental (si/no) y analizar cada componente de pufa con los puntajes del P-ECOHIS.

\section{RESULTADOS}

En mayor porcentaje fueron las madres las acompañantes de los niños (97\%), las cuales tuvieron una edad promedio de $32,67 \pm 7,58$ años, con nivel secundario como grado de instrucción (51\%) y un ingreso familiar de una RMV (41\%). Los niños tuvieron una edad promedio de 4,18 $\pm 0,96$ años y en mayor proporción fueron de sexo masculino (57 \%) (Tabla I).

La media del puntaje total del P-ECOHIS fue de $19,98 \pm 6,9$, con un puntaje mínimo de 6 y un máximo de 41 , la prevalencia de las lesiones cariosas no tratadas y lesiones cariosas extensas en dentina fue de 90 $\%$ y $71 \%$, con un puntaje medio de $4,25 \pm 3,16$ y 2,59 $\pm 2,62$ respectivamente. El dolor dental fue reportado en $68,7 \%$ de los niños y el $24,7 \%$ de ellos tuvieron al menos una extracción dental producto de una lesión cariosa que no fue tratada. Los valores de pufa y sus componentes se resumen en la Tabla II, donde también se observa que el $77 \%$ de los dientes cariados no tratados desarrollaron una infección oral.

En la Tabla III se observa que solo el dolor dental tuvo una asociación estadísticamente significativa con el puntaje del P-ECOHIS $(p<0,05)$, la probabilidad de que los niños con dolor dental tengan puntaje alto en el P-ECOHIS es el cuádruple que los niños que no experimentaban dolor $(\mathrm{OR}=3,76 ; 95 \%$ IC: 1,61-8,80). Al evaluar los componentes de pufa, los niños que presentaron compromiso pulpar (OR=2,44; $95 \%$ IC: $1,12-5,30)$ y presencia de fístula (OR=4,39; $95 \%$ IC: $1,72-11,24)$ tuvieron altas probabilidades de tener altos puntajes en el $\mathrm{P}$ ECOHIS que los niños que no están afectados por esas condiciones $(p<0,05)$ (Tabla IV).

La ulceración, absceso (consecuencias clínicas de caries dental no tratada), así como la lesión cariosa extensa en dentina e historia de extracción dental, no presentaron asociación con el puntaje PECOHIS. 
MUNAYCO-PANTOJA, E. R.; PEREYRA-ZALDÍVAR, H. \& CADILLO-IBARRA, M. M. Consecuencias clínicas de las lesiones cariosas no tratadas y su impacto en la calidad de vida de preescolares peruanos. Int. J. Odontostomat., 15(1):256-262, 2021

Tabla I. Características sociodemográficas de los participantes del estudio.

\begin{tabular}{llcc}
\hline Características demográficas & & $\mathrm{n}$ & $\%$ \\
\hline Sexo acompañante & Femenino & 145 & 97 \\
Sexo niño & Masculino & 5 & 3 \\
& Femenino & 65 & 43 \\
Edad del acompañante & Masculino & 85 & 57 \\
Edad del niño & $20-32$ & 77 & 51 \\
& $33-54$ & 73 & 49 \\
& 3 años & 42 & 28 \\
Ingreso familiar por mes* & 4 años & 31 & 21 \\
& 5 años & 77 & 51 \\
Grado de instrucción del acompañante & <1RMV & 49 & 33 \\
& RMV & 61 & 41 \\
& Primaria & 40 & 26 \\
& Secundaria & 71 & 8 \\
& Superior & 62 & 51 \\
\end{tabular}

*RMV - remuneración mínima vital

Tabla II. Media, desviación estándar y frecuencia de pufa y sus componentes.

\begin{tabular}{llll}
\hline & $\mathrm{p}$ & $\mathrm{OR}$ & $95 \% \mathrm{IC}$ \\
\hline Caries extensa en dentina & 0,65 & 1,43 & $0,31-6,70$ \\
pufa & 0,43 & 2,05 & $0,35-11,94$ \\
Dolor dental & $0,002^{*}$ & 3,76 & $1,61-8,80$ \\
Historia de extracción & 0,76 & 1,14 & $0,49-2,64$
\end{tabular}

*desviación estándar.

Tabla III. Resultados del análisis de regresión logística binaria de la caries extensa en dentina, pufa, dolor dental e historia de extracción y la calidad de vida relacionada a la salud oral usando PECOHIS.

\begin{tabular}{llll}
\hline & $\mathrm{p}$ & $\mathrm{OR}$ & $95 \% \mathrm{IC}$ \\
\hline Caries extensa en dentina & 0,65 & 1,43 & $0,31-6,70$ \\
PUFA & 0,43 & 2,05 & $0,35-11,94$ \\
Dolor dental & $0,002^{*}$ & 3,76 & $1,61-8,80$ \\
Historia de extracción & 0,76 & 1,14 & $0,49-2,64$
\end{tabular}

${ }^{*} p<0,05$ - estadísticamente significativo, OR: odds ratio, IC: intervalo de confianza.

Tabla IV. Resultados del análisis de regresión logística binaria de las consecuencias clínicas de caries no tratadas y la calidad de vida relacionada a la salud oral usando $\mathrm{P}$-ECOHIS

\begin{tabular}{llll}
\hline & $\mathrm{p}$ & $\mathrm{OR}$ & $95 \% \mathrm{IC}$ \\
\hline Compromiso pulpar & $0,025^{*}$ & 2,44 & $1,12-5,30$ \\
Ulceración por traumatismo & 0,30 & 1,61 & $0,65-4,01$ \\
Fistula & $0,002^{*}$ & 4,39 & $1,72-11,24$ \\
Absceso & 0,86 & 0,92 & $0,39-2,21$
\end{tabular}

${ }^{*} p<0,05$ - estadísticamente significativo, OR: odds ratio, IC: intervalo de confianza.

\section{DISCUSIÓN}

La prevalencia de caries no tratada fue del 90 $\%$ ligeramente mayor que la reportada por Monse et al., y mucho más alta que los estudios realizados en niños preescolares nigerianos (Oziegbe \& Esan, 2013), indios (Mehta \& Bhalla) y brasileros (Ramos-Jorge et al., 2014). Existe variedad de índices y criterios utilizados para medir la prevalencia de caries; por ejemplo, en la última edición de la encuesta de salud oral de la Organización mundial de la salud (OMS), una lesión cariosa profunda con afectación pulpar generalmente se considera bajo el código "caries de dentina" y la afectación pulpar no se menciona en absoluto en el sistema de puntuación de caries (World Health Organization, 2013). Si solo se presentan datos con el índice ceod a los encargados de la toma de decisiones en salud, no serían conscientes de la verdadera condición dental en la población, ya que si no existe un registro oportuno de la caries no tratada pueden derivar en infecciones odontogénicas, porque ambas están correlacionadas (Grund et al., 2015).

La experiencia pufa se determina como el radio pufa-caries no tratada, nos indica el porcentaje de dientes con caries que desarrollaron infecciones odontogénicas a nivel poblacional y la prevalencia pufa, nos indica el porcentaje de las piezas afectadas por las consecuencias clínicas de la caries no tratada (Monse et al.). Ambas nos ayudan a que los criterios se uniformicen y los resultados puedan ser comparables. En esta investigación el resultado para prevalencia fue $77 \%(3,19 \pm 3,1)$ y es superior a lo informado en estudios previos (Figueiredo et al., 2011; Leal et al., 2012; Ferraz et al., 2014) pero similar al resultado informado por Monse et al. (84 \%) para niños filipinos. En población peruana, los valores publicados por otros autores han sido más altos (Morales \& Gómez, 2019), al parecer existe una necesidad de tratamiento que aún no han sido abordada por los responsables de las políticas en salud oral y es evidente que está no es prioridad para el sistema de salud peruano.

El valor medio de pufa encontrado fue 3,19 \pm 3,1 , un valor más alto que lo encontrado en otros países $(0,1 \pm 0,5)$ (Grund et al.), $(0,4 \pm 0,9)$ (Figueiredo et al.), $(1,03 \pm 1,65)$ (Sudan et al., 2018) y $(1,18 \pm 0,57)$ (Kamran et al., 2017), por lo que se deduciría que exis- 
ten diferentes patrones de infecciones odontogénicas alrededor del mundo; si es comparado con estudios peruanos, el valor es menor al presentado por Morales \& Gómez. Lo que, si es repetitivo, en todos los estudios evaluados, es la alta prevalencia del componente "p" (compromiso pulpar) comparado con los demás componentes. En algunos estudios los componentes "u", "f" o "a" tienen el valor de cero (Grund et al.; Sudan et al.), motivo por el cual diferentes autores han propuesto la modificación del índice pufa (Frencken et al., 2011).

Murthy et al. (2014) sugieren que los componentes "f" y "a"' pueden agruparse juntos ya que se refieren al mismo proceso inflamatorio, pero en diferentes etapas, además el tratamiento requerido seria el mismo: tratamiento de endodoncia o extracción, aunque ellos se basan en un estudio realizado en población adolescente. También fue sugerido por Figueiredo et al., donde evaluaron población de 5 a 6 años. Cabe resaltar que el índice de pufa no fue diseñado para servir como un índice de necesidad de tratamiento, sino para cuantificar la gravedad de caries dental no tratada y para evaluar la presencia de infecciones odontogénicas (Holmgren et al., 2014). Aun así, se requieren más estudios para realizar tales modificaciones (Mehta \& Bhalla).

En este estudio, el dolor dental impacta negativamente en la CVRSB, lo cual ha sido confirmado por otros autores (Clementino et al., 2015b) independientemente de la edad (Fernandes et al., 2019) o el cuestionario utilizado para medirlo (Freire et al., 2018), por lo que debe prevenirse y tratarse urgentemente, al igual que las consecuencias clínicas de lesiones cariosas no tratadas que también impactan en los niños y las familias. Por otro lado, en esta investigación, las lesiones cariosas extensas en dentina no impactan en la CVRSB, como si lo refieren otros autores (Leal et al.; Corrêa-Faria et al.). Estos hallazgos hacen una contribución importante a la toma de decisiones clínicas y establecimiento de prioridades en atención pública de la salud oral.

La pregunta directa a los cuidadores sobre el dolor dental infantil, es una de las limitantes del estudio, ya que esto puede conducir a una subestimación de la prevalencia, debido a que estos solo recuerdan episodios de mayor intensidad de dolor $u$ ocasiones donde la actividad diaria del niño se vio interrumpida (Ramos-Jorge et al., 2013), además, pueden estar influenciados por un sesgo de memoria (Ferraz et al.). Evaluar el dolor dental en niños pequeños es una tarea difícil incluso para los dentistas experimentados y es dependiente de las percepciones de los cuidadores (Freire et al.), por lo que se recomienda utilizar cuestionarios dirigidos a estos.

En conclusión, las lesiones cariosas extensas en dentina no impactan en la CVRSB de los preescolares peruanos, como si impacta negativamente, el compromiso pulpar y la presencia de fistula como consecuencias clínicas de lesiones cariosas no tratadas. Por lo que, nuestros resultados enfatizan la necesidad de reforzar la importancia de la prevención y tratamiento de la caries en la dentición decidua; y la necesidad de mejorar la provisión de servicios de salud oral a la población infantil. Lograr que el país implemente el resultado de esta investigación dentro de las políticas de salud basadas en evidencia y que estas disminuyan la carga de la enfermedad para que esta sea una carga innecesaria para la sociedad peruana.

MUNAYCO-PANTOJA, E. R.; PEREYRA-ZALDÍVAR, H. \& CADILLO-IBARRA, M. M. Clinical consequences of untreated carious lesions and their impact on the quality of life of peruvian preschoolers. Int. J. Odontostomat. 15(1):256-262, 2021.

ABSTRACT: Untreated dental caries has repercussions on the health of children who suffer from it, it also affects family well-being; Furthermore, there is a greater probability of presenting clinical consequences, which would aggravate the health condition. The objective of the study was to investigate the impact of extensive carious lesions on dentin and the clinical consequences of untreated carious lesions on the quality of life of Peruvian preschoolers. A total of 150 children, 3 to 5 years old, were evaluated using ICDAS and PUFA. The dental extraction history was also evaluated and the presence of dental pain was recorded. The impact of oral health on quality of life was evaluated using the Peruvian version of ECOHIS (PECOHIS). The binary logistic regression model was used to analyze the association between the prevalence of extensive carious lesions in dentin, PUFA, extraction history and dental pain with the P-ECOHIS score. The mean total P-ECOHIS score was $19.98 \pm 6.9$, the prevalence of untreated carious lesions and extensive carious lesions in dentin was $90 \%$ and $71 \%$, respectively. $77 \%$ of untreated carious teeth developed an oral infection. Dental pain had a statistically significant association with the P-ECOHIS score $(p<0.05)(\mathrm{OR}=3.76$; $95 \% \mathrm{Cl}: 1.61$ - 8.80). Children who had pulp involvement (OR $=2.44 ; 95 \%$ $\mathrm{Cl}: 1.12-5.30)$ and the presence of fistula (OR $=4.39 ; 95 \% \mathrm{Cl}$ : $1.72-11.24)$ were highly likely to have high scores on the PECOHIS ( $p$ <0.05). Extensive carious lesions in dentin do not impact the quality of life of Peruvian preschoolers, as it does negatively impact, pulpal involvement and the presence of fistula as clinical consequences of untreated carious lesions. health. 


\section{REFERENCIAS BIBLIOGRÁFICAS}

Allen, P. F. Assessment of oral health related quality of life. Health. Qual. Life Outcomes, 1, 40, 2003,

Arrow, P. \& Klobas, E. Evaluation of the early childhood oral health impact scale in an Australian preschool child population. Aust. Dent. J., 60(3):375-81, 2015.

Clementino, M. A.; Gomes, M. C.; Pinto-Sarmento, T. C.; Martins, C. C.; Granville-Garcia, A. F. \& Paiva, S. M. Perceived impact of dental pain on the quality of life of preschool children and their families. PloS One, 10(6):e0130602, 2015a.

Clementino, M. A.; Pinto-Sarmento, T. C.; Costa, E. M.; Martins, C. C.; Granville-Garcia, A. F. \& Paiva, S. M. Association between oral conditions and functional limitations in childhood. J. Oral Rehabil., 42(6):420-9, 2015b.

Corrêa-Faria, P.; Daher, A.; Freire, M.; de Abreu, M.; Bönecker, M. \& Costa, L. R. Impact of untreated dental caries severity on the quality of life of preschool children and their families: a cross-sectional study. Qual. Life Res., 27(12):3191-8, 2018.

Duijster, D.; Sheiham, A.; Hobdell, M. H.; Itchon, G. \& Monse, B. Associations between oral health-related impacts and rate of weight gain after extraction of pulpally involved teeth in underweight preschool filipino children. BMC Public Health, 13:533, 2013

Fernandes, I. B.; Souto-Souza, D.; Primo-Miranda, E. F.; Marques, L. S.; Ramos-Jorge, M. L. \& Ramos-Jorge, J. Perceived impact of dental pain on the quality of life of children aged 1-3 years and their families. Eur. Arch. Paediatr. Dent., 20(6):557-63, 2019.

Ferraz, N. K.; Nogueira, L. C.; Pinheiro, M. L.; Marques, L. S.; Ramos-Jorge, M. L. \& Ramos-Jorge, J. Clinical consequences of untreated dental caries and toothache in preschool children. Pediatr. Dent., 36(5):389-92, 2014.

Figueiredo, M. J.; de Amorim, R. G.; Leal, S. C.; Mulder, J. \& Frencken, J. E. Prevalence and severity of clinical consequences of untreated dentine carious lesions in children from a deprived area of Brazil. Caries Res., 45(5):435-42, 2011.

Freire, M.; Corrêa-Faria, P. \& Costa, L. R. Effect of dental pain and caries on the quality of life of Brazilian preschool children. Rev. Saude Publica, 52:30, 2018.

Frencken, J. E.; de Amorim, R. G.; Faber, J. \& Leal, S. C. The Caries Assessment Spectrum and Treatment (CAST) index: rational and development. Int. Dent. J., 61(3):117-23, 2011.

Grund, K.; Goddon, I.; Schüler, I. M.; Lehmann, T. \& HeinrichWeltzien, R. Clinical consequences of untreated dental caries in German 5- and 8-year-olds. BMC Oral Health, 15(1):140, 2015.

Holmgren, C.; van Palenstein Helderman, W.; Monse, B.; HeinrichWeltzien, R. \& Benzian, H. Modifications to the PUFA index: are they justified at this stage? Med. Princ. Pract., 23(3):292-3, 2014.

ICCMSTM Caries Management. International Caries Classification and Management System. ICDAS A simple, logical, evidencebased system for detection and classification of caries in dental education, clinical practice, dental research, and dental public health. ICCMSTM Caries Management, 2020. Disponible en: https://www.iccms-web.com/content/icdas

Kamran, R.; Farooq, W.; Faisal, M. R. \& Jahangir, F. Clinical consequences of untreated dental caries assessed using PUFA index and its covariates in children residing in orphanages of Pakistan. BMC Oral Health, 17(1):108, 2017.

Kassebaum, N. J.; Bernabé, E.; Dahiya, M.; Bhandari, B.; Murray, C. J. \& Marcenes, W. Global burden of untreated caries: a systematic review and metaregression. J. Dent. Res., 94(5):650-8, 2015.
Kassebaum, N. J.; Smith, A.; Bernabé, E.; Fleming, T. D.; Reynolds, A. E.; Vos, T.; Murray, C.; Marcenes, W. \& GBD 2015 Oral Health Collaborators. Global, regional, and national prevalence, incidence, and disability-adjusted life years for oral conditions for 195 countries, 1990-2015: a systematic analysis for the global burden of diseases, injuries, and risk factors. J. Dent. Res., 96(4):380-7, 2017.

Leal, S. C.; Bronkhorst, E. M.; Fan, M. \& Frencken, J. E. Untreated cavitated dentine lesions: impact on children's quality of life. Caries Res., 46(2):102-6, 2012,

Locker, D. \& Allen, F. What do measures of 'oral health-related quality of life' measure?. Community Dent. Oral Epidemiol., 35(6):401-11, 2007.

López Ramos, R. P.; García Rupaya, C. R.; Villena-Sarmiento, R. \& Bordoni, N. E. Cross cultural adaptation and validation of the Early Childhood Health Impact Scale (ECOHIS) in Peruvian preschoolers. Acta Odontol. Latinoam., 26(2):60-7, 2013,

Mehta, A. \& Bhalla, S. Assessing consequences of untreated carious lesions using PUFA index among 5-6 years old school children in an urban Indian population. Indian J. Dent. Res., 25(2): 150-3, 2014

Monse, B.; Heinrich-Weltzien, R.; Benzian, H.; Holmgren, C. \& van Palenstein Helderman, W. PUFA--an index of clinical consequences of untreated dental caries. Community Dent. Oral Epidemiol., 38(1):77-82, 2010.

Morales, M. L. \& Gómez, G. W. Caries dental y sus consecuencias clínicas relacionadas al impacto en la calidad de vida de preescolares de una escuela estatal. Rev. Estomatol. Herediana, 29(1):17-29, 2019.

Murthy, A. K.; Pramila, M. \& Ranganath, S. Prevalence of clinical consequences of untreated dental caries and its relation to dental fear among 12-15-year-old schoolchildren in Bangalore city, India. Eur. Arch. Paediatr. Dent., 15(1):45-9, 2014.

Nalliah, R. P.; Allareddy, V.; Elangovan, S.; Karimbux, N. \& Allareddy, V. Hospital based emergency department visits attributed to dental caries in the United States in 2006. J. Evid. Based Dent. Pract., 10(4):212-22, 2010.

Nora, Â. D.; da Silva Rodrigues, C.; de Oliveira Rocha, R.; Soares, F.; Minatel Braga, M. \& Lenzi, T. L. Is Caries Associated with Negative Impact on Oral Health-Related Quality of Life of Preschool Children? A Systematic Review and Meta-Analysis. Pediatr. Dent., 40(7):403-11, 2018.

Oziegbe, E. O. \& Esan, T. A. Prevalence and clinical consequences of untreated dental caries using PUFA index in suburban Nigerian school children. Eur. Arch. Paediatr. Dent., 14(4):227-31, 2013,

Peres, M. A.; Macpherson, L.; Weyant, R. J.; Daly, B.; Venturelli, R.; Mathur, M. R.; Listl, S.; Celeste, R. K.; Guarnizo-Herreño, C. C.; Kearns, C.; et al. Oral diseases: a global public health challenge. Lancet, 394(10194):249-60, 2019.

Petersen P. E. Global policy for improvement of oral health in the 21 st century--implications to oral health research of World Health Assembly 2007, World Health Organization. Community Dent. Oral Epidemiol., 37(1):1-8, 2009.

Petersen P. E. The World Oral Health Report 2003: continuous improvement of oral health in the 21st century--the approach of the WHO Global Oral Health Programme. Community Dent. Oral Epidemiol., 31 Suppl. 1:3-23, 2003

Piva, F.; Pereira, J. T.; Luz, P. B.; Hugo, F. N. \& de Araújo, F. B. Caries progression as a risk factor for increase in the negative impact on OHRQOL-a longitudinal study. Clin. Oral Investig., 22(2):819-28, 2018.

Ramos-Jorge, J.; Alencar, B. M.; Pordeus, I. A.; Soares, M. E.; Marques, L. S.; Ramos-Jorge, M. L. \& Paiva, S. M. Impact of dental caries on quality of life among preschool children: emphasis on the type of tooth and stages of progression. Eur. J. Oral Sci., 123(2):88-95, 2015. 
MUNAYCO-PANTOJA, E. R.; PEREYRA-ZALDÍVAR, H. \& CADILLO-IBARRA, M. M. Consecuencias clínicas de las lesiones cariosas no tratadas y su impacto en la calidad de vida de preescolares peruanos. Int. J. Odontostomat., 15(1):256-262, 2021.

Ramos-Jorge, J.; Pordeus, I. A.; Ramos-Jorge, M. L.; Marques, L. S. \& Paiva, S. M. Impact of untreated dental caries on quality of life of preschool children: different stages and activity. Community Dent. Oral Epidemiol., 42(4):311-22, 2014.

Ramos-Jorge, M. L.; Ramos-Jorge, J.; Mota-Veloso, I.; Oliva, K. J.; Zarzar, P. M. \& Marques, L. S. Parents' recognition of dental trauma in their children. Dent. Traumatol., 29(4):266-71, 2013,

Seirawan, H.; Faust, S. \& Mulligan, R. The impact of oral health on the academic performance of disadvantaged children. Am. J. Public Health, 102(9):1729-34, 2012,

Selwitz, R. H.; Ismail, A. I. \& Pitts, N. B. Dental caries. Lancet, 369(9555):51-9, 2007.

Sudan, J.; Sogi, G. M. \& Veeresha, L. K. Assessing clinical sequelae of untreated caries among 5-, 12-, and 15-year-old school children in ambala district: A cross-sectional study. J. Indian Soc. Pedod. Prev. Dent., 36(1):15-20, 2018.

World Health Organization (WHO). Oral Health Surveys-Basic Methods. $5^{\text {th }}$ ed. Ginebra, World Health Organization, 2013.
Dirección para correspondencia:

Evelyn R. Munayco-Pantoja

Avenida Venezuela 1288 Breña

Lima

PERÚ

E-mail: evelyn.munayco@unmsm.edu.pe 